Article

\title{
Terahertz Single-Pixel Imaging Improved by Using Silicon Wafer with $\mathrm{SiO}_{2}$ Passivation
}

\author{
Rongbin She ${ }^{1,2} \mathbb{D}$, Wenquan Liu ${ }^{1}$, Guanglu Wei ${ }^{1,2}$ and Yuanfu Lu ${ }^{1, *(\mathbb{D})}$ and Guangyuan Li ${ }^{1, *(D)}$ \\ 1 Shenzhen Institutes of Advanced Technology, Chinese Academy of Sciences, Shenzhen 518055, China; \\ rb.she@siat.ac.cn (R.S.); wq.liu@siat.ac.cn (W.L.); gl.wei@siat.ac.cn (G.W.) \\ 2 Shenzhen College of Advanced Technology, University of Chinese Academy of Sciences, \\ Shenzhen 518055, China \\ * Correspondence: yf.lu@siat.ac.cn (Y.L.); gy.li@siat.ac.cn (G.L.)
}

Received: 3 March 2020; Accepted: 31 March 2020; Published: 2 April 2020

check for updates

\begin{abstract}
We demonstrate terahertz single-pixel imaging is improved by using a photomodulator based on silicon passivated with $\mathrm{SiO}_{2}$. By exploring various $\mathrm{SiO}_{2}$ thicknesses, we show that the modulation factor of the as-fabricated terahertz photomodulator can reach 0.9 , three times that based on bare silicon. This improvement originates from chemical passivation, as well as anti-reflection. Single-pixel imaging experiments based on the compressed sensing method show that reconstructed images adopting the new photomodulator have better quality than the conventional terahertz modulator based on bare silicon. Since the passivation process is routine and low cost, we expect this work will reduce the cost of terahertz photomodulator and single-pixel THz imaging, and advance their applications.
\end{abstract}

Keywords: terahertz single-pixel imaging; passivation; photomodulation; compressed sensing

\section{Introduction}

Terahertz (THz) imaging has potential applications in a variety of fields, such as non-destructive detection [1,2], security inspection [3,4], and biomedicine [5,6]. Despite great progress, commercially available terahertz focal plane array detectors are still too expensive for most researchers. Therefore, terahertz imaging techniques based on single-pixel detectors have been the focus to date. Among these techniques, computational sampling methods are attractive due to their efficiency compared with the slow mechanical scanning method.

As the key for THz single-pixel imaging based on computational sampling methods, terahertz modulation techniques have received increasing attention. In 2008, Chan et al. [7] adopted standard printed circuit boards as the random matrix to modulate the terahertz transmission, and demonstrated a THz single-pixel imaging by further combining the compressed sensing (CS) algorithm. In order to avoid the use of moving masks, which are difficult to align, Watts et al. [8] made use of a metamaterial spatial modulator, Sensale-Rodriguez et al. [9] employed arrays of graphene electro-absorption modulators, and Kannegulla et al. [10] and Shams et al. [11] demonstrated photo-induced coded-aperture imaging using a semiconductor illuminated by a commercial available digital light processing projector. Recently, silicon wafers have been widely used as $\mathrm{THz}$ photomodulators, based on which Stantchev et al. [12,13] demonstrated THz superresolution imaging. She et al. [14] used graphene on silicon as the THz photomodulator and demonstrated $\mathrm{THz}$ single-pixel imaging based on the Fourier space spectrum. While various approaches have been adopted to boost photomodulation [15-17], there has been less focus on the semiconducting photoconductive materials themselves. Quite recently, Hooper et al. [18] demonstrated that a passivated silicon wafer can 
greatly improve the efficiency of $\mathrm{THz}$ photomodulation. However, $\mathrm{THz}$ single-pixel imaging based on such a THz photomodulator has not been reported to date.

In this work, we demonstrate that $\mathrm{THz}$ single-pixel imaging based on compressed sensing algorithm can be greatly enhanced by $\mathrm{THz}$ photomodulators based on silicon passivated with $\mathrm{SiO}_{2}$. We will first show that $\mathrm{THz}$ photomodulators based on passivated silicon can have a much higher modulation factor than one based on bare silicon. The effects of the $\mathrm{SiO}_{2}$ thickness on the photomodulation improvement and the underlying physics will be discussed. Imaging quality based on the as-fabricated $\mathrm{THz}$ photomodulators will be compared with the conventional one based on bare silicon.

\section{Experimental Setup}

Figure 1 illustrates the light-controlled $\mathrm{THz}$ modulation and single-pixel imaging system based on a home-built THz time-domain spectroscopy (THz-TDS) system [19]. A pair of biased low-temperature-grown GaAs (LT-GaAs) photoconductive antennas, which are illuminated by femtosecond laser pulses $(\lambda=800 \mathrm{~nm},<100 \mathrm{fs}, 80 \mathrm{MHz}$; Coherent Vitesse 800-5), are used to generate and detect $\mathrm{THz}$ radiations. Collimated $\mathrm{THz}$ wave with a diameter of $3.82 \mathrm{~mm}$ is directed onto a high-resistance silicon wafer $(2000 \Omega \cdot \mathrm{cm}, 500 \mu \mathrm{m}$ thick), both surfaces of which are passivated with $\mathrm{SiO}_{2}$. The passivation is realized following a high-temperature thermal oxidation process that is routine in the field of solar cells [20]. The transmitted THz wave is then collected by a single-pixel $\mathrm{THz}$ detector. The beam profile (size $4 \mathrm{~mm} \times 4 \mathrm{~mm}$ ) of an $808 \mathrm{~nm}$ linearly polarized (the polarization is parallel to the optical platform) continuous-wave (CW) laser is first patterned by a digital micromirror device (DMD). The DMD, detached from DLP3000 (Texas Instruments), has $608 \times 684$ micro-mirrors, each of which has a pitch of $7.6 \mu \mathrm{m}$ and behaves as a binary reflector with the maximum frame rate reaching $4000 \mathrm{~Hz}$. The laser beam is then projected onto the passivated silicon. Unless otherwise specified, all the experiments in this work were performed with a high laser power of $330 \mathrm{~mW}$. When the laser spot reflected by the DMD is changed from black to white, the THz transmittance through the passivated silicon decreases from a high level to a low level, i.e., from " 1 " to " 0 ". For terahertz single-pixel imaging based on compressed sensing (CS) approach [7,11,21], the target, such as a cartwheel patterned in a gold film, is deposited on top of the front $\mathrm{SiO}_{2}$ layer.

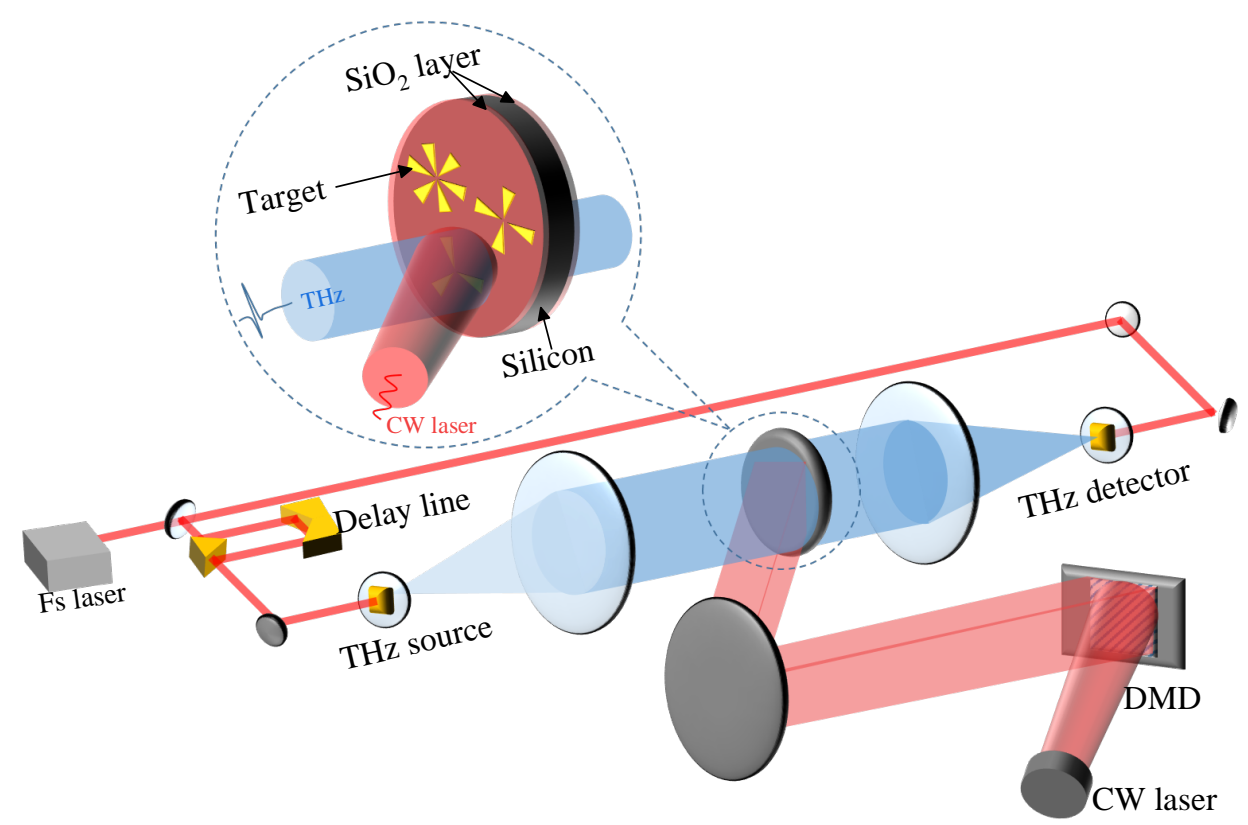

Figure 1. Schematic of the experimental setup for the $\mathrm{THz}$ single-pixel imaging. The $\mathrm{CW}$ laser is reflected on the modulator by DMD, and target objects are cartwheels patterned in gold film, which is deposited on the modulator substrate. 
For a THz image of $N \times N$ pixels to be measured, a series of masks are projected onto the target. The cumulative signal is collected using a single-pixel detector. A series of such measurements with the total number of $M$ lead to $[7,21]$

$$
Y^{\mathrm{CS}}=P^{\mathrm{CS}} X
$$

where $Y^{\mathrm{CS}}$ is an $M \times 1$ vector of measurements, $X$ is the image with $N \times N$ pixels ordered in a $N^{2} \times 1$ vector, and $P^{\mathrm{CS}}$ denotes the series (with the number of $\mathrm{M}$ ) of $N \times N$ projection masks $p_{N \times N}^{\mathrm{CS}}$ ordered in an $M \times N^{2}$ matrix. For each measurement, the projection mask $p_{N \times N}^{\mathrm{CS}}$ can be generated with Hadamard coding, which consists of " 1 " and " -1 " elements with each row orthogonal to all other rows [22]. In experiments, the Hadamard projection mask $P^{\mathrm{CS}}$ composed of " 1 " and " -1 " elements is replaced by the substation of two masks $P^{\mathrm{CS}, \mathrm{I}}$ and $P^{\mathrm{CS}, \mathrm{II}}$, both of which consist of " 1 " and " 0 " elements [12], i.e.,

$$
P^{\mathrm{CS}}=P^{\mathrm{CS}, \mathrm{I}}-P^{\mathrm{CS}, \mathrm{II}},
$$

Therefore, $Y^{\mathrm{CS}}$ in Equation (1) should be replaced by $Y^{\mathrm{CS}}=Y^{\mathrm{CS}, \mathrm{I}}-Y^{\mathrm{CS}, \mathrm{II}}$, where $Y^{\mathrm{CS}, \mathrm{I} / \mathrm{II}}=$

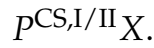

Using the CS approach, the number of measurements for assembling the image can be much smaller than the number of image pixels that is $M \ll N^{2}$. The sampling ratio is defined as

$$
S_{\mathrm{R}} \equiv M / N^{2}
$$

Even with highly undersampled measurement sets, the image can still be accurately reconstructed. In order to reconstruct the $\mathrm{THz}$ image, we adopted an algorithm that performs total variation minimization, and that has large signal-to-noise ratio [12,13],

$$
\min \left\|P^{\mathrm{CS}} X-Y^{\mathrm{CS}}\right\|_{2}-\gamma \cdot \operatorname{TV}(\mathrm{X}), \quad \text { where } \operatorname{TV}(\mathrm{X})=\sum_{i} \sqrt{\left(D_{\mathrm{h}} X\right)_{i}^{2}+\left(D_{\mathrm{V}} X\right)_{i}^{2}} .
$$

Here $\gamma$ is a relaxation variable that determines the smoothness of the reconstructed image, and $D_{\mathrm{h}}$ and $D_{\mathrm{v}}$ are the discretized gradient operators along the horizontal and vertical directions, respectively.

\section{THz Photomodulation Experiments}

To investigate the effects of the $\mathrm{SiO}_{2}$ passivation thickness, various thicknesses were prepared. This was achieved by etching the double-sided passivated silicon wafer, where the thickness of each $\mathrm{SiO}_{2}$ layer was measured to be $287 \mathrm{~nm}$. The passivated silicon wafer was first cut into small pieces of the same size, and was then etched by using $40 \%$ potassium hydroxide solution with different duration time. The thickness of the $\mathrm{SiO}_{2}$ layer was measured by a film thickness meter. Table 1 summarizes the obtained $\mathrm{SiO}_{2}$ thicknesses of each side (both sides have the same thickness).

Table 1. As-fabricated silicon wafer with different $\mathrm{SiO}_{2}$ passivation thicknesses.

\begin{tabular}{cccccccccc}
\hline Sample & S1 & S2 & S3 & S4 & S5 & S6 & S7 & S8 & S9 \\
\hline Thickness (nm) & 0 & 21 & 109 & 140 & 148 & 180 & 216 & 247 & 287 \\
\hline
\end{tabular}

The THz time-domain signal transmitted through the sample under $\mathrm{CW}$ laser illumination $E_{\text {samp }}(t)$ was collected. As a reference, the $\mathrm{THz}$ time-domain signal without any modulator $E_{\text {ref }}(t)$ was collected. The THz transmittance is then calculated by [23],

$$
T_{\mathrm{on}}(\omega)=\frac{E_{\mathrm{samp}}(\omega)}{E_{\mathrm{ref}}(\omega)},
$$

where $\omega$ is the frequency of the transmitted THz wave, $E_{\text {samp }}(\omega)$ and $E_{\text {ref }}(\omega)$ are the Fourier transform of $E_{\mathrm{samp}}(t)$ and $E_{\text {ref }}(t)$, respectively. 
Figure 2a shows that as the $\mathrm{SiO}_{2}$ thickness increases from $0 \mathrm{~nm}$ to $148 \mathrm{~nm}$, the transmittance of the $\mathrm{THz}$ field decreases from about $55 \%$ to $7 \%$. Further increasing the $\mathrm{SiO}_{2}$ thickness results in increased transmittance: for the thickness of $287 \mathrm{~nm}$, the transmittance is $30 \%$.
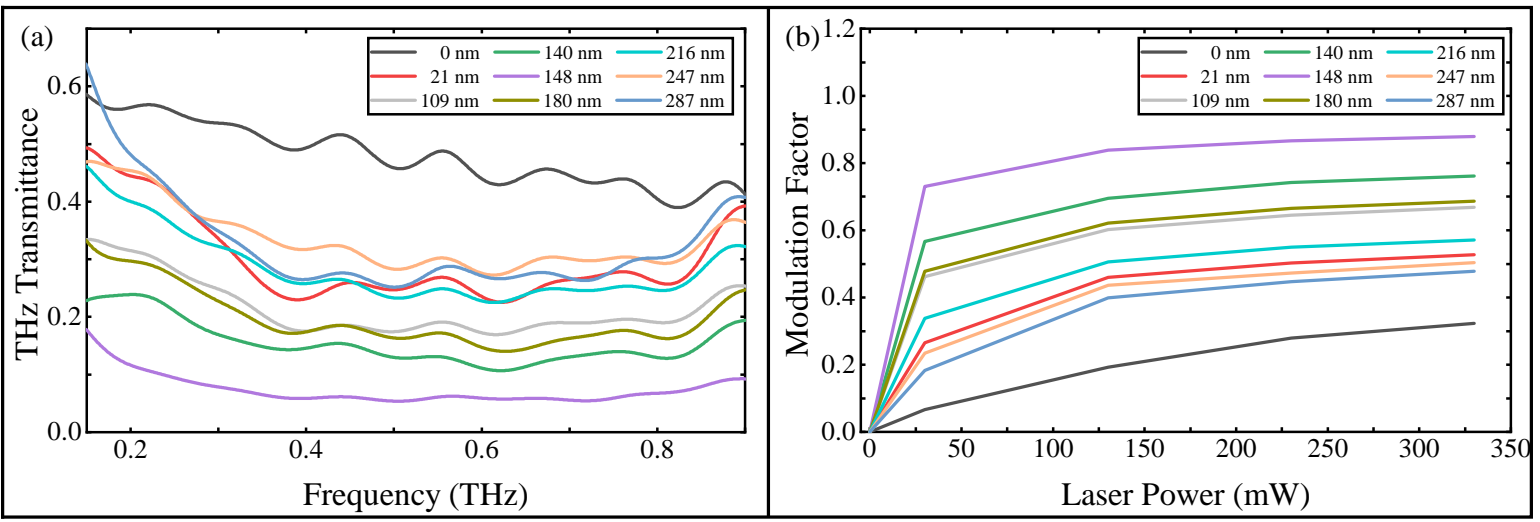

Figure 2. (a) $\mathrm{THz}$ transmittance for different $\mathrm{SiO}_{2}$ thicknesses. (b) Modulation factor versus $\mathrm{CW}$ laser power.

To evaluate the modulation efficiency of different samples, we make use of the modulation factor (MF), which is defined as the change of the integrated transmitted $\mathrm{THz}$ power under the illumination [24],

$$
\mathrm{MF} \equiv \frac{\int T_{\text {off }}(\omega) \mathrm{d} \omega-\int T_{\text {on }}(\omega) \mathrm{d} \omega}{\int T_{\text {off }}(\omega) \mathrm{d} \omega}
$$

Here, $T_{\text {off }}(\omega)$ and $T_{\text {on }}(\omega)$ are the transmitted powers when the laser is off and on, respectively. Figure $2 \mathrm{~b}$ shows that all the MFs for different $\mathrm{SiO}_{2}$ thicknesses increase with the $\mathrm{CW}$ laser power, where " $0 \mathrm{~nm}$ " implies the bare silicon wafer. This is because the carrier density in the semiconductor is proportional to the optical power of the laser beam and is modulated through photodoping [25]. Strikingly, for laser power of $330 \mathrm{~mW}$ the maximum MF of 0.9 is achieved by sample S5 with $\mathrm{SiO}_{2}$ thickness of $148 \mathrm{~nm}$; this value is three times of that for the bare silicon (MF $=0.3$ for sample S1). Figure $3 \mathrm{a}$ shows that as the $\mathrm{SiO}_{2}$ thickness increases, the MFs for different $\mathrm{CW}$ laser powers first increase and then decrease, exhibiting maximum values around the $\mathrm{SiO}_{2}$ thickness of $148 \mathrm{~nm}$.
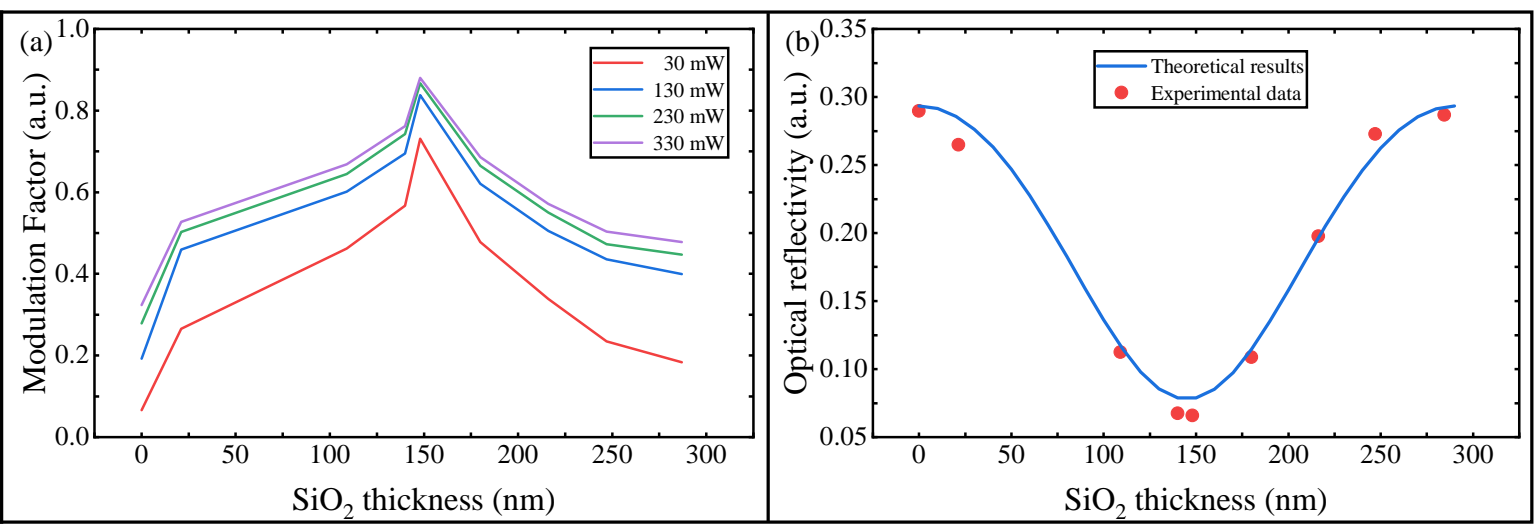

Figure 3. (a) $\mathrm{THz}$ modulation factor versus $\mathrm{SiO}_{2}$ thickness under the illumination of different $\mathrm{CW}$ laser powers. (b) Calculated (blue curve) and measured (red dots) optical reflectivity of the CW laser versus $\mathrm{SiO}_{2}$ thickness.

To understand the physics origin for the terahertz photomodulation performance, we measured the optical reflectivity of the CW laser by the as-fabricated samples. In experiments, the optical reflectivity was obtained by dividing the power reflected from the sample by that from a gold mirror. Figure $3 \mathrm{~b}$ shows that as the $\mathrm{SiO}_{2}$ thickness increases, the optical reflectivity first decreases from about 
$30 \%$ to about $6 \%$, and then increases again to $30 \%$. The smallest optical reflectivity of $6 \%$ is achieved for $\mathrm{SiO}_{2}$ thickness of $147 \mathrm{~nm}$. The experimental data agree well with the theoretical calculation following the Fresnel formulas, where the incidence angle is $25^{\circ}$, and the refractive indices of $\mathrm{SiO}_{2}$ and silicon at the wavelength of $808 \mathrm{~nm}$ are taken to be 1.45 and 3.68, respectively. The optimal thickness of $\mathrm{SiO}_{2}$ as well as the corresponding minimum optical reflectivity may vary as the incident angle changes. Specifically, for smaller incidence angles, the minimum optical reflectivity can be slightly smaller; for the Brewster angle between the air- $\mathrm{SiO}_{2}$ interface $\left(\theta_{\mathrm{B}}=55.4^{\circ}\right)$, the optical reflectivity of the $\mathrm{CW}$ laser keeps at $13 \%$ irrespective of the $\mathrm{SiO}_{2}$ thickness. The independence of the optical reflectivity on the $\mathrm{SiO}_{2}$ thickness at $\theta_{\mathrm{B}}$ is due to full transmission through the air- $\mathrm{SiO}_{2}$ interface in this scenario, thus the optical reflectivity is exactly that by the $\mathrm{SiO}_{2}-\mathrm{Si}$ interface. Although even lower optical reflectivity at small incidence angles can result in even larger MF, in our experiments the specific angle of $25^{\circ}$ was used due to space limitation of the experimental setup.

Figure 3 shows that as the $\mathrm{SiO}_{2}$ thickness increases, the $\mathrm{MF}$ of the $\mathrm{THz}$ radiation and the optical reflectivity of the $\mathrm{CW}$ laser behave reversely. When the $\mathrm{SiO}_{2}$ thickness increases from $0 \mathrm{~nm}$ to the $148 \mathrm{~nm}$, the optical reflectivity decreases, thus the concentration of generated carriers raises and the $\mathrm{THz}$ transmittance reduces since more photons impinge onto the silicon wafer producing carriers. When the $\mathrm{SiO}_{2}$ thickness further increases, the optical reflectivity increases, reducing the photons that can be absorbed by the silicon wafer, the concentration of generated carriers, and the $\mathrm{THz}$ transmittance. These suggest the key role played by the anti-reflection effect of $\mathrm{SiO} 2$.

However, for samples S1 and S9, both of which have almost the same optical reflectivity, as shown by Figure 3, the former has much smaller terahertz MF than the latter. This is because the dielectric layer added on top of the silicon surface terminates dangling bonds that arise due to the lattice termination, resulting in a reduction in trap density [18]. Therefore, we show that the $\mathrm{SiO}_{2}$ not only plays a role of chemical passivation, but also acts as an anti-reflection film that enhances the use of light energy [26].

\section{THz Single-Pixel Imaging Experiments}

We now study the improvement of the THz single-pixel imaging quality by using the as-fabricated $\mathrm{THz}$ photo-controlled modulators based on passivated silicon wafers. Three targets were prepared: three-arm, four-arm and six-arm cartwheels.

To quantify the image quality, we adopt signal-to-noise ratio (SNR) as the figure of merit following refs $[13,27,28]$. It is defined as the average value of intensity in the bright region, $\mu$ (signal), divided by the standard deviation of the noise in the signal-free area of the image, $\sigma$ (background), that is $[13,28]$

$$
\mathrm{SNR} \equiv \frac{\mu(\text { signal })}{\sigma(\text { background })}
$$

Figure 4 compares the reconstructed terahertz images by using two photomodulators made of the passivated silicon wafer with the highest MF and the conventional bare silicon wafer, i.e., samples S5 and S1. These terahertz images were obtained under the same experimental conditions: the laser power is $330 \mathrm{~mW}$, and the sampling ratio is $100 \%$. Results show that for the bare silicon wafer, the reconstructed images have very poor quality due to the small MF of 0.3 , so that the SNR is not available; however, for the passivated silicon wafer with the highest MF of 0.9 , the reconstructed image is very clean and clear, and the corresponding SNRs are about 1.0. In other words, the terahertz single-pixel imaging is greatly improved by replacing the conventional photomodulator based on the bare silicon wafer with the new one based on the passivated silicon wafer. 


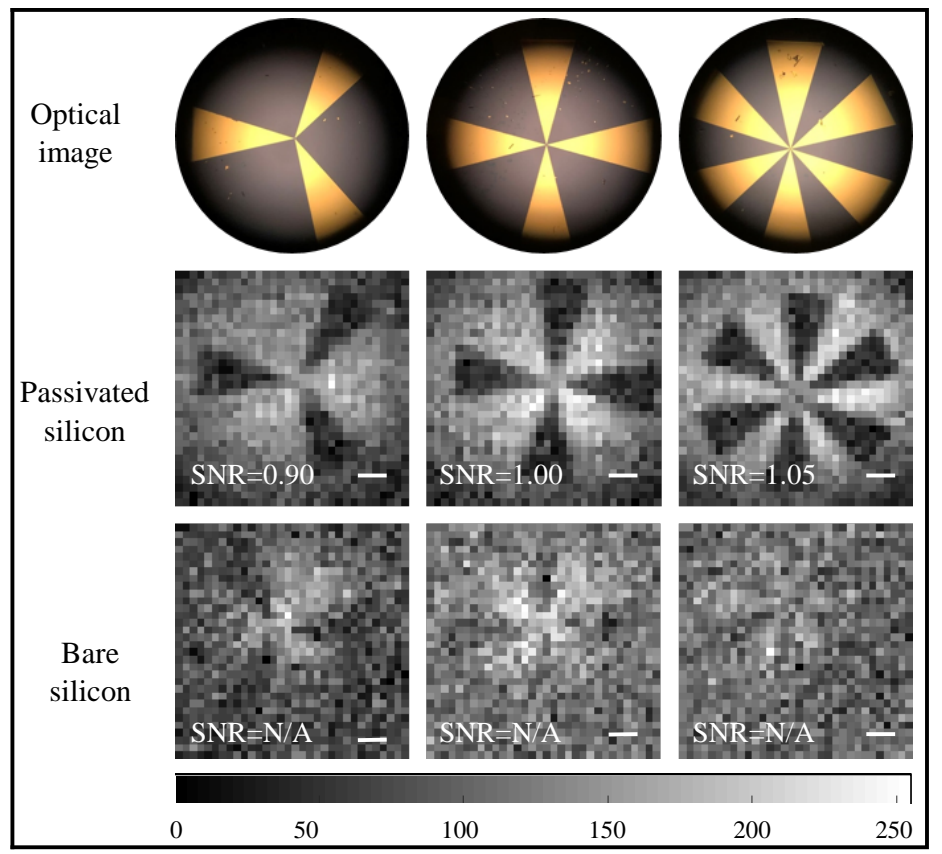

Figure 4. Top panel: optical images of three targets. Middle and bottom panels: reconstructed $32 \times 32$ terahertz images $(N=32)$ based on photomodulators made of passivated and bare silicon wafers, i.e., samples S5 and S0, respectively. The images were reconstructed under full sampling. The scalar bars denote $500 \mu \mathrm{m}$.

By using the photomodulator made of sample S5, Figure 5 shows that the quality of reconstructed images increases with the sampling ratio in general. The change trend of the SNR with the sampling ratio also confirms this phenomenon, i.e., as the sampling ratio increases, the sharpness of the image gradually increases. The image can be well reconstructed only for sampling ratios of more than $25 \%$. For a sampling ratio of $25 \%$, there still exists pronounced background noise. The noise is greatly suppressed as the sampling ratio increases.

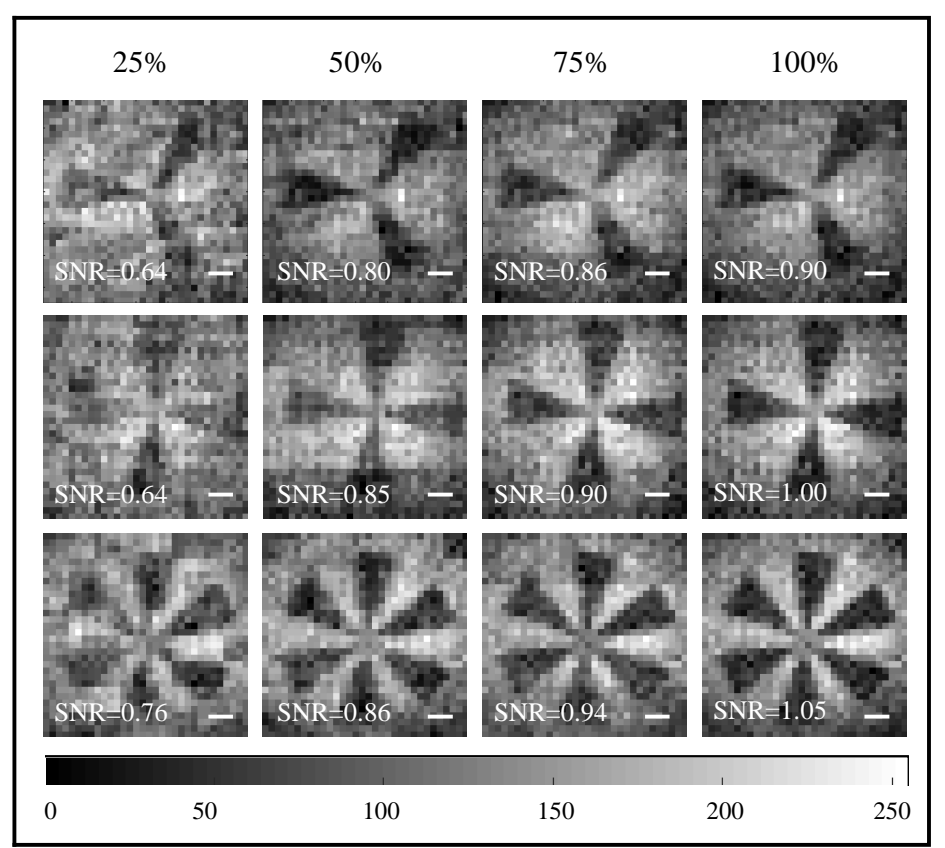

Figure 5. Reconstructed $32 \times 32$ terahertz images under different sampling ratios: $25 \%, 50 \%, 75 \%$ and $100 \%$. Scalar bars denote $500 \mu \mathrm{m}$. 


\section{Conclusions}

In conclusion, we have demonstrated that $\mathrm{THz}$ photomodulation and single-pixel imaging is improved by using $\mathrm{SiO}_{2}$ passivated silicon. By exploring different $\mathrm{SiO}_{2}$ thicknesses, we have found that for laser illumination power of $330 \mathrm{~mW}$, the largest MF of 0.9 is achieved at a thickness of $148 \mathrm{~nm}$, for which the optical reflectivity reaches the minimum. This MF is three times the MF of bare silicon wafer. We have shown that this great improvement originates from chemical passivation and optical anti-reflection that are both introduced by the $\mathrm{SiO}_{2}$ passivation. $\mathrm{THz}$ single-pixel imaging experiments based on the CS approach have shown that by using the conventional $\mathrm{THz}$ photomodulator based on the bare silicon wafer, the reconstructed image had very poor quality; however, by using a greatly improved photomodulator based on the passivated silicon wafer, the reconstructed image is very clean and clear. We have further shown that by using the new photomodulator, sampling ratios above $25 \%$ can result in single-pixel $\mathrm{THz}$ imaging with acceptable quality. This can greatly reduce the imaging time. Since surface passivation of silicon with $\mathrm{SiO}_{2}$ is efficient, we expect our work will advance and promote the development of applications of $\mathrm{THz}$ photomodulation and single-pixel imaging.

Author Contributions: Conceptualization, R.S., W.L. and Y.L.; methodology, R.S., W.L. and G.W.; software, R.S.; validation, W.L., G.L. and Y.L.; formal analysis, R.S.; investigation, R.S.; resources, Y.L.; data curation, R.S.; writing-original draft preparation, R.S.; writing-review and editing, R.S. and G.L.; visualization, R.S.; supervision, G.L. and Y.L.; project administration, Y.L.; funding acquisition, G.L. and Y.L. All authors have read and approved the final version of the manuscript.

Funding: This research was funded by the Shenzhen Research Foundation (Grant Nos. JCYJ20160608153308846, JCYJ20170413152328742, JCYJ20180507182444250), National Key Research and Development Program of China (No. 2017YFC0803506), and Youth Innovation Promotion Association of the Chinese Academy of Sciences (No. 2016320).

Conflicts of Interest: The authors declare no conflict of interest.

\section{References}

1. Duling, I.; Zimdars, D. Terahertz imaging: Revealing hidden defects. Nat. Photonics 2009, 3, 630. [CrossRef]

2. Jepsen, P.U.; Cooke, D.G.; Koch, M. Terahertz spectroscopy and imaging-Modern techniques and applications. Laser Photon. Rev. 2011, 5, 124. [CrossRef]

3. Shen, Y.C.; Lo, T.; Taday, P.F.; Cole, B.E.; Tribe, W.R.; Kemp, M.C. Detection and identification of explosives using terahertz pulsed spectroscopic imaging. Appl. Phys. Lett. 2005, 86, 241116. [CrossRef]

4. Skvortsov, L.A. Standoff Detection of Hidden Explosives and Cold and Fire Arms by Terahertz Time-Domain Spectroscopy and Active Spectral Imaging (Review). J. Appl. Spectrosc. 2014, 81, 725. [CrossRef]

5. Zhong, H.; Redo-Sanchez, A.; Zhang, X.C. Identification and classification of chemicals using terahertz reflective spectroscopic focal-plane imaging system. Opt. Express 2006, 14, 9130. [CrossRef]

6. Shen, Y.C. Terahertz pulsed spectroscopy and imaging for pharmaceutical applications: A review. Int. J. Pharm. 2011, 417, 48. [CrossRef]

7. Chan, W.L.; Moravec, M.L.; Baraniuk, R.G.; Mittleman, D.M. Terahertz imaging with compressed sensing and phase retrieval. Opt. Lett. 2008, 33, 974. [CrossRef]

8. Watts, C.M.; Shrekenhamer, D.; Montoya, J.; Lipworth, G.; Hunt, J.; Sleasman, T.; Krishna, S.; Smith, D.R.; Padilla, W.J. Terahertz compressive imaging with metamaterial spatial light modulators. Nat. Photonics 2014, 8, 605. [CrossRef]

9. Sensale-Rodriguez, B.; Rafique, S.; Yan, R.; Zhu, M.; Protasenko, V.; Jena, D.; Liu, L.; Xing, H.G. Terahertz imaging employing graphene modulator arrays. Opt. Express 2013, 21, 2324. [CrossRef]

10. Kannegulla, A.; Jiang, Z.; Rahman, S.M.; Shams, M.I.B.; Fay, P.; Xing, H.G.; Cheng, L.J.; Liu, L. Coded-Aperture Imaging Using Photo-Induced Reconfigurable Aperture Arrays for Mapping Terahertz Beams. IEEE Trans. Terahertz Sci. Technol. 2014, 4, 321. [CrossRef]

11. Shams, M.I.B.; Jiang, Z.; Rahman, S.; Qayyum, J.; Cheng, L.J.; Xing, H.G.; Fay, P.; Liu, L. Approaching real-time terahertz imaging with photo-induced coded apertures and compressed sensing. Electron. Lett. 2014, 50, 801. [CrossRef] 
12. Stantchev, R.I.; Sun, B.; Hornett, S.M.; Hobson, P.A.; Gibson, G.M.; Padgett, M.J.; Hendry, E. Noninvasive, near-field terahertz imaging of hidden objects using a single-pixel detector. Sci. Adv. 2016, 2, e1600190. [CrossRef]

13. Stantchev, R.I.; Phillips, D.B.; Hobson, P.; Hornett, S.M.; Padgett, M.J.; Hendry, E. Compressed sensing with near-field THz radiation. Optica 2017, 4, 989. [CrossRef]

14. She, R.; Wenquan, L.; Lu, Y.; Zhou, Z.; Li, G. Fourier single-pixel imaging in the terahertz regime. Appl. Phys. Lett. 2019, 115, 021101. [CrossRef]

15. Weis, P.; Garcia-Pomar, J.L.; Höh, M.; Reinhard, B.; Brodyanski, A.; Rahm, M. Spectrally Wide-Band Terahertz Wave Modulator Based on Optically Tuned Graphene. ACS Nano 2012, 6, 9118. [CrossRef]

16. Cao, Y.; Gan, S.; Geng, Z.; Liu, J.; Yang, Y.; Bao, Q.; Chen, H. Optically tuned terahertz modulator based on annealed multilayer $\mathrm{MoS}_{2}$. Sci. Rep. 2016, 6, 22899. [CrossRef]

17. Yang, D.S.; Jiang, T.; Cheng, X.A. Optically controlled terahertz modulator by liquid-exfoliated multilayer $\mathrm{WS}_{2}$ nanosheets. Opt. Express 2017, 25, 16364. [CrossRef]

18. Hooper, I.R.; Grant, N.E.; Barr, L.E..; Hornett, S.M.; Murphy, J.D.; Hendry, E. High efficiency photomodulators for millimeter wave and THz radiation. Sci. Rep. 2019, 9, 18304. [CrossRef]

19. Liu, W.Q.; Lu, Y.F.; Jiao, G.H.; Chen, X.F.; Li, J.Y.; Chen, S.H.; Dong, Y.M.; Lv, J.C. Terahertz optical properties of the cornea. Opt. Commun. 2016, 359, 344. [CrossRef]

20. Zhao, J.; Wang, A.; Green, M.A. 24.5\% Efficiency Silicon PERT Cells on MCZ Substrates and 24.7\% Efficiency PERL Cells on FZ Substrates. Prog. Photovolt Res. Appl. 1999, 7, 471. [CrossRef]

21. Chan, W.L.; Charan, K.; Takhar, D.; Kelly, K.F.; Baraniuk, R.G.; Mittleman, D.M. A single-pixel terahertz imaging system based on compressed sensing. Appl. Phys. Lett. 2008, 93, 121105. [CrossRef]

22. Valova, I.; Kosugi, Y. Hadamard-based image decomposition and compression. IEEE Trans. Inf. Technol. Biomed. 2000, 4, 306. [CrossRef] [PubMed]

23. Neu, J.; Schmuttenmaer, C.A. Tutorial: An introduction to terahertz time domain spectroscopy (THz-TDS). J. Appl. Phys. 2018, 124, 231101. [CrossRef]

24. Wang, G.; Zhang, B.; Ji, H.; Liu, X.; He, T.; Lv, L.; Hou, Y.; Shen, J. Monolayer graphene based organic optical terahertz modulator. Appl. Phys. Lett. 2017, 110, 023301. [CrossRef]

25. Shrekenhamer, D.; Watts, C.M.; Padilla, W.J. Terahertz single pixel imaging with an optically controlled dynamic spatial light modulator. Opt. Express 2013, 21, 012507. [CrossRef]

26. Blakers, A.W.; Wang, A.; Milne, A.M.; Zhao, J.; Green, M.A. 22.8\% efficient silicon solar cell. Appl. Phys. Lett. 1989, 55, 1363. [CrossRef]

27. Chen, S.C.; Du, L.H.; Meng, K.; Li, J.; Zhai, Z.H.; Shi, Q.W.; Li, Z.R.; Zhu, L.G. Terahertz wave near-field compressive imaging with a spatial resolution of over $\lambda / 100$. Opt. Lett. 2019, 44, 21. [CrossRef]

28. Shchelokova, A.V.; Slobozhanyuk, A.P.; Melchakova, I.V.; Glybovski, S.B.; Webb, A.G.; Kivshar, Y.S.; Belov, P.A. Locally Enhanced Image Quality with Tunable Hybrid Metasurfaces. Phys. Rev. Appl. 2018, 9, 014020. [CrossRef] 